\title{
Intrathecal Baclofen for Severe Spasticity: Longitudinal Data From the Product Surveillance Registry
}

\author{
Mya Caryn Schiess, MD, FANA*; Sam Eldabe, MB, ChB, FRCA ${ }^{\dagger}{ }^{\circ}$ \\ Peter Konrad, MD, PhD; ; Lisa Molus, MB, ChB, FRCA ${ }^{+}$; \\ Robert Spencer, MS, MBA ${ }^{\mathbb{s}}$; Katherine Stromberg, MS $^{\S}$; Todd Weaver, PhD $^{\S}$; \\ Robert Plunkett, MD"
}

\begin{abstract}
Objective: To assist in the assessment of intrathecal baclofen (ITB) therapy risks and benefits by providing surgical intervention rate, safety, and elective device replacement rate data.

Materials and Methods: An ongoing prospective, long-term, multicenter Product Surveillance Registry (PSR) (NCT01524276) enrolled consented patients implanted with the SynchroMed II infusion system. Pump and catheter performance data were collected, with patients followed prospectively for events related to the device, procedure, and therapy. Investigators provided event descriptions, patient symptoms, and patient outcomes.

Results: We analyzed registry data from 1743 patients (77\% adult, 46.8\% female) treated with ITB for severe spasticity at 53 registry sites between August 2003 and October 2017, for an accumulated 6481 patient-years. Discontinuation from the registry was largely (58.6\% of discontinued patients) due to study site closure and patient relocation; exit due to an adverse event was limited to $0.3 \%$. After 10 years, $87.2 \%$ of adult and $76.3 \%$ of pediatric patients continued with ITB. Overall, $99.1 \%$ of pumps reaching end of battery life were replaced at the time of explant.

Conclusions: ITB therapy for the treatment of severe spasticity requires surgical implantation of a programmable infusion system for chronic drug delivery. If complications arise, many necessitate surgical intervention for correction. For spinal and cerebral spasticity in pediatric and adult patients, discontinuation rates due to an adverse event were low (0.3\%), and there was high acceptance $(99.1 \%)$ of surgical intervention for therapy continuation. Patient/caregiver willingness to accept surgical and other risks for therapy continuation was extremely high.
\end{abstract}

Keywords: intrathecal baclofen, registry, risk, spasticity

Conflict of Interest: The Product Surveillance Registry is maintained by Medtronic, Inc. Authors Spencer, Stromberg, and Weaver are employees of Medtronic. The other authors received financial support for this research from Medtronic. The author(s) report receiving financial support for the research. Funding for the Product Surveillance Registry, data monitoring, statistical analysis, and medical writing were provided by Medtronic, Inc.

\section{INTRODUCTION}

Severe spastic tone can be the most disabling consequence of a neurologic insult that creates an upper motor neuron lesion from either a cerebral (e.g., cerebral palsy, acute brain injury, stroke) or spinal (e.g., spinal cord injury, multiple sclerosis) etiology. Spasticity is clinically recognized as a disorder of sensory-motor control brought about by an imbalance of supraspinal descending excitatory/inhibitory neurons that regulate muscle tone (1).

Treatment has focused on improving function, mobility, and range of motion to alleviate pain and prevent secondary complications. Conventional medical management (CMM) involves physical therapy and antispasmodic medications, with baclofen the most frequently used. The effectiveness of oral baclofen is often limited by its systemic side effects of dizziness, sedation, confusion, lethargy, and muscle weakness (2). Intrathecal baclofen (ITB) administration delivers drug directly to receptor sites, reducing
Address correspondence to: Mya Caryn Schiess, Department of Neurology, UTHMS, Houston, TX 77030, USA.

Email: mya.c.schiess@uth.tmc.edu

* Department of Neurology, UTHMS, Houston, TX, USA;

+ The James Cook University Hospital, Middlesbrough, UK;

₹ Vanderbilt University Medical Center, Nashville, TN, USA;

$\S$ Medtronic, Minneapolis, MN, USA; and

" Jacobs School of Medicine and Biomedical Sciences, University at Buffalo, Department of Neurosurgery, Roswell Park Comprehensive Cancer Center, Buffalo, NY, USA

For more information on author guidelines, an explanation of our peer review process, and conflict of interest informed consent policies, please go to http:// www.wiley.com/WileyCDA/Section/id-301854.html

This is an open access article under the terms of the Creative Commons Attribution-NonCommercial License, which permits use, distribution and reproduction in any medium, provided the original work is properly cited and is not used for commercial purposes. 
drug dosage, and potentially improving tolerability. ITB is indicated for treating severe spasticity of cerebral/spinal origin that has failed to respond to maximum tolerated or recommended doses of oral antispasmodics (3).

Despite the approval of ITB for treatment of spasticity by the US Food and Drug Administration (1992 Spinal; 1996 Cerebral) and UK National Health Service commissioning (2013), it has not attracted as much interest as other neuromodulation procedures. A study in the United Kingdom identified a gap between the actual number of implantations ( 3 per million population) and the estimated need for ITB therapy for spasticity (4.6-5.7 per million population) (4). One possible explanation is concern about the risk profile of implantable infusion systems, especially perceptions regarding complications necessitating surgical intervention. Whether treated with ITB or CMM, patients with severe spasticity require attentive management and frequent interventions. In a retrospective claims analysis by Saulino et al., however, patients with severe spasticity receiving ITB therapy had fewer inpatient facility costs, fewer emergency department visits, less physiotherapy, fewer office visits, fewer injections and reduced prescription drug costs than patients receiving CMM (5).Thus, by health economics and outcomes research (HEOR) measures, ITB improved quality of life and was cost-effective, a conclusion reached by other similar studies $(6,7)$.

The objective of this article was to provide data on ITB therapy risks and insights on patient benefit through the use of a large registry and reported surgical intervention rates along with therapy durability/system replacement rates.

\section{MATERIALS AND METHODS}

We analyzed data from the Product Surveillance Registry (PSR) to assess the safety (adverse events or AEs related to the device, implantation, or infusion therapy), durability (duration of pump implants and patient survival from study exit), and patient satisfaction (elective pump replacement) with ITB therapy for spasticity. The PSR comprises the largest, multicenter cohort of ITB-treated patients worldwide, covering an accumulated 6481 patient-years of ITB experience.

\section{Registry Description}

The Medtronic Implantable Systems Performance Registry (ISPR; ClinicalTrials.gov Identifier: NCT01524276), initiated in 2003, is described in detail by Konrad et al. (8). Results presented there include data collected under the ISPR protocol, which focused on a single manufacturer's product performance at a number of implant centers around the world in which longitudinal patient follow-up was highly likely for the life of the implant. In 2013, the registry expanded data collection with a corresponding name change to the PSR. The PSR platform was designed to conduct ongoing nonrandomized, active prospective post-market surveillance under a common protocol, with specific appendices for neuromodulation products/therapies, by enrolling patients with an eligible product-in this case, implanted ITB pumps. Prior to patient enrollment, all sites obtained Ethics Committee/Institutional Review Board approval to allow tracking of the device performance in each consented patient. The PSR sites contributing to these data are noted in the Acknowledgments section.

\section{Patients}

Potential PSR patients were identified from the practices of participating physicians as meeting the specific indication of severe spasticity for the SynchroMed II infusion system (Medtronic, Inc. Minneapolis, MN, USA) and were enrolled at initial implant or at the time of pump replacement; excluded were patients enrolled/ implanted for the treatment of chronic nonmalignant pain and cancer pain. The patient or legally authorized representative provided written authorization and/or consent per institution and geographical requirements prior to data collection. Patients inaccessible for follow-up, excluded per local law, or currently enrolled in or planning to enroll in any concurrent drug and/or device study that may confound results were excluded. Data were only included for patients who consent to enroll. After enrollment, patients were followed longitudinally per standard of care, with status updates obtained every 6 months with no predefined duration. Data for all global registry centers and all patients enrolled are reported.

\section{Data Collection}

The registry was created to monitor the performance of the implanted infusion systems, but the types of data collected have evolved over time. Safety data were originally focused on devicerelated events but expanded in 2010 to include all serious AEs and all events related to implanted or external components of the infusion system, the implant procedure, or the infusion therapy. Reporting of device-related surgical intervention, however, has remained consistent throughout the course of the registry. Collection of outcome data by registry sites (i.e., EQ-5D, numerical pain rating scale) was added to the PSR in 2013; however, a spasticity or tone assessment was not added. Elective pump replacement was assessed here as a surrogate for patient satisfaction. All data were entered by site personnel in a centralized database.

\section{Analytic Methods}

Data included in this analysis were collected from August 2003 through October 31, 2017 from patients in the ongoing PSR who were treated with ITB for severe spasticity. Summary statistics are presented either as percentages for categorical variables or as mean (standard deviation, SD; or minimum/maximum) for continuous values.

In order to evaluate the ongoing risk faced by ITB patients, the rate of surgical interventions by year post-implant was calculated as follows: number of nonbattery replacement surgical interventions per number of active patients within each year post-implant for the subset of patients who were enrolled in the registry with their initial infusion system.

Long-term therapy retention was assessed using time-to-event Kaplan-Meier survival estimates. Patients with their initial implant at the time of enrollment were included in the analysis. Study exit due to an $\mathrm{AE}$ or device event or due to an inactive system (i.e., therapy abandoned for greater than 6 months or system explanted without replacement) was the event of interest. Survival time (i.e., duration of therapy retention) was defined as months from implant to therapy discontinuation. Patients who were active in the registry or who exited from the registry due to nontherapy discontinuation reasons were censored at their last visit.

\section{RESULTS}

A total of 7975 pump patients were enrolled at 64 sites across the US, Europe, and Latin America, with 1743 (46.8\% female) from 53 sites being treated for severe spasticity. The majority of patients with spasticity $(77 \%, 1350 / 1743)$ were at least 18 years 
old at enrollment. Most of the pediatric patients (patients under 18 years old at enrollment) were implanted for the treatment of cerebral palsy (CP, 78\%, 307/393). Among adults, the most common treatment indication was multiple sclerosis (MS, 33\%, 446/1350), followed by spinal cord injury (SCl, 17\%, 231/1350). Figure 1 provides spasticity etiology for adult and pediatric patients. At the time of enrollment, 72\% (1255/1743) of patients were implanted with their initial pump and were naïve to infusion therapy. The remaining $28 \%(488 / 1743)$ of patients were enrolled with a pump replacement. For patients with an initial implant, the average age for the adult cohort was 46 years $( \pm 14,18-86)$ and for children 10 years $( \pm 4,2-18)$.

Patient participation within this registry is not defined by any endpoint date or duration of follow-up. Patients remained in the registry until they were no longer available for follow-up (e.g., study site closure, lost to follow-up, change to another treating institution or health-care provider) or their continued participation is no longer warranted (e.g., system explant or therapy discontinuation). Over time there have been 1202 spasticity patients $(69 \%)$ who discontinued from the registry, with the reasons for study exit for these patients in Table 1. "Registry study site closure" (29.7\%) and "Patient care transferred" (28.9\%) account for $704(58.6 \%)$ of these discontinuations, with "Lost to

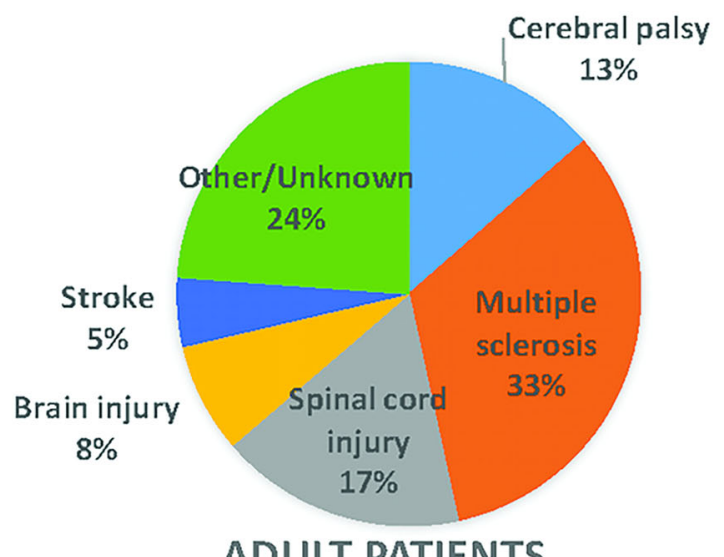

ADULT PATIENTS

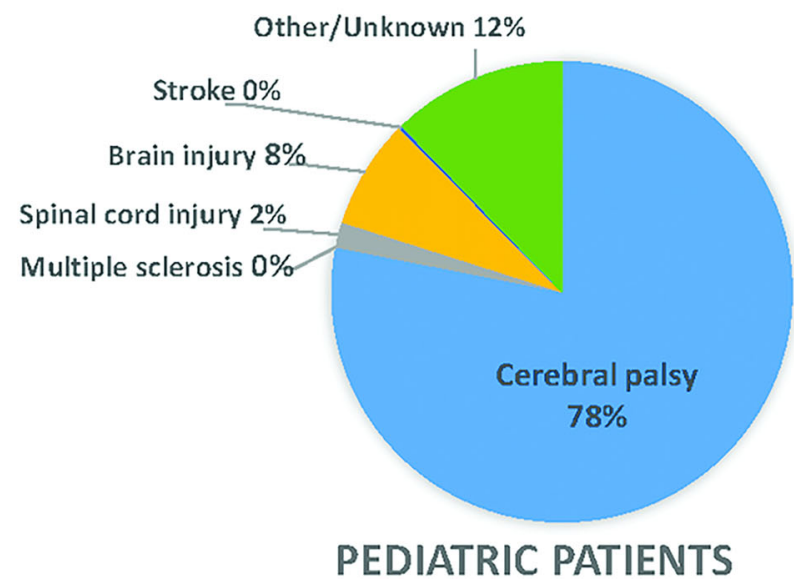

Figure 1. Spasticity etiology by adult/pediatric patients. [Color figure can be viewed at wileyonlinelibrary.com] follow-up" and "Patient no longer available for follow-up" accounting for another 112 (9.3\%) patients. Therapy events (e.g., surgical interventions) for these 816 enrolled patients were included through the date of their registry discontinuation, but final outcome remains unknown. Overall, the average duration of patient follow-up was 44.6 months $(S D=40.2)$ and ranged from 0 months to more than 13 years, with an accumulated follow-up of 6481 patient-years. Unlike studies with a predefined finite duration, this registry has been active for more than 16 years and continues to follow existing patients and enroll new patients.

Table 2 summarizes the number of surgical device interventions (i.e., pump or catheter explant or surgical procedure to modify an implanted component, such as pump repositioning or catheter revision) by year postimplantation for patients who enrolled with an initial implant in the registry (therapy naïve). A count of the number of interventions, the number of patients (therapy naïve) who were active during each year, and the rate of intervention/ patient was summarized by age group and overall. The table excludes pump explants due to normal battery depletion, which had no associated AE. Each period reported included interventions occurring prior to the last year reported, that is, Year 0-1 included surgical interventions that occurred before completing the first year of implant, Year 1-2 included surgical interventions that occurred on or after 1 year of implant, but prior to the second year of implant, and so on.

Study exit reasons were used as a surrogate indicator of patient satisfaction with ITB therapy. A patient who discontinued from the registry due to an adverse or device event or due to an inactive system was considered a discontinuation from the registry due to dissatisfaction with the therapy. Patient survival from therapy-related discontinuation is summarized for adult and pediatric patients in Fig. 2. Within the cohort of patients receiving an initial pump implant at enrollment, $87.2 \%$ (95\% confidence interval [Cl]: $83.4 \%, 90.1 \%)$ of adult patients and $76.3 \%$ (95\% Cl: $68.6 \%$, $82.4 \%$ ) of pediatric patients maintained ITB therapy (i.e., had not discontinued from the registry for reasons related to adverse or device events or therapy discontinuation) through at least 10 years. Patient survival from therapy-related discontinuation is shown by spasticity indication in Fig. 2 .

Implanted, battery powered pumps eventually require replacement due to battery depletion. In the case of the SynchroMed II infusion system, the designed performance life is capped at 84 months, with a typical time to replacement of 75 months for the currently available infusion system. The clinician-programmer displays an anticipated end of service (EOS) date with each pump interrogation; and an elective replacement indicator (ERI) message is displayed 90 days in advance of the EOS. This lead time for a necessary surgical intervention to continue ITB therapy, in contrast to the more acute decisions needed in cases of system complications, allows for a thorough patient/caregiver assessment of therapy benefits in deciding whether to undergo the replacement procedure. A total of 426 patients progressed to the point of at least one elective pump replacement (462 total pumps: 364 in ITB naïve patients, 98 in patients enrolled with a pump replacement) within the registry; 32 patients had undergone more than one pump replaced while enrolled in the registry. Mean implant duration (months) is summarized in Table 3 for the 462 pumps explanted due to a normal battery depletion. More than $99 \%$ of the 462 pumps that were explanted due to normal battery depletion were replaced on the same day, with four patients (4/364 ITB naïve at enrollment) electing system explant; two due to loss of therapeutic benefit and one each due to "patient request" and 
Table 1. Study Exit Reasons.

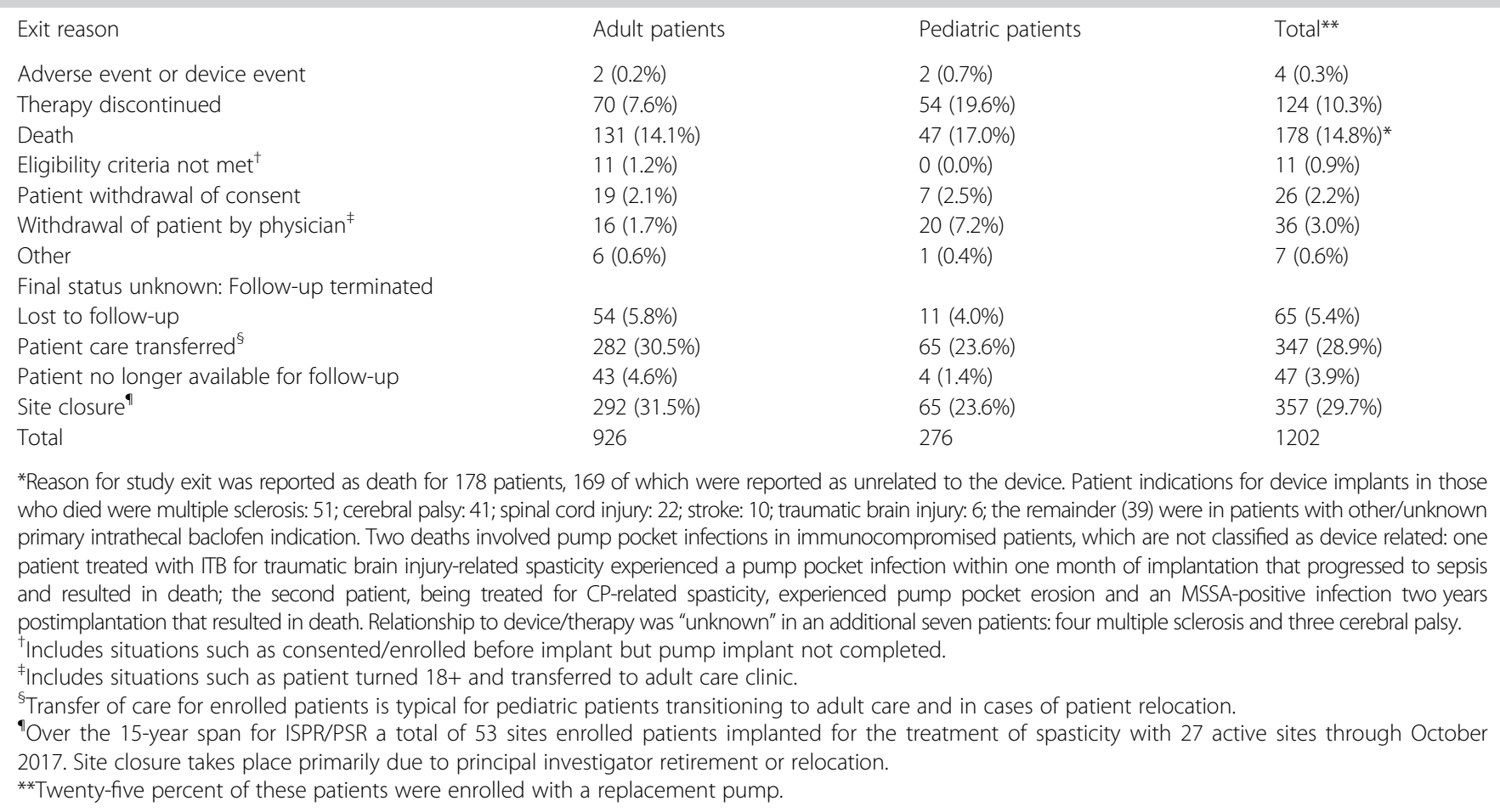

"unacceptable follow-up and maintenance requirements" (Table 3).

\section{DISCUSSION}

Our safety analysis included 1743 patients with average duration of patient follow-up of 44.6 months $(S D=40.2$ ) that ranged from 0 months to more than 13 years, and an accumulated 6481 patientyears of experience. The number of surgical interventions per year post-implant for therapy naïve patients was highest in the first year (113 total) followed by year 6-7 (63 total) and year 3-4 (42 total). Durability analysis found that mean implant duration was 72 months. More than $99 \%$ of the pumps explanted due to normal battery depletion were replaced on the same day, demonstrating that patients/families or their physicians elected to continue therapy. Long-term therapy retention remained high even after 10 years, whereby $87.2 \%$ of adult and $76.3 \%$ of pediatric patients continued with ITB therapy.

ITB therapy was originally evaluated for the treatment of severe spasticity of spinal origin $(9,10)$, with indications expanded to include severe spasticity of cerebral origin, including cerebral palsy (11), brain injury (12), and stroke (13-15). Previous studies have demonstrated efficacy in spasticity of multiple origins, with long-term benefits (11,16-18). A 1997 meta-analysis of 27 studies with 490 ITB-treated patients included spasticity due to $\mathrm{SCl}$ (42\% of patients), MS (33\%), CP (12\%), and other causes (13\%) (19). The results demonstrated significant decreases in mean Ashworth and spasm scores in each diagnostic group at 18 months average follow-up; $8 \%$ of implanted patients discontinued treatment by 1 year in these early studies.

More recently, Creamer et al. compared the effectiveness of ITB therapy with CMM in 60 subjects with post-stroke spasticity in a randomized multicenter study (15). In addition to improvements in

Table 2. Surgical Interventions by Year Postimplant, by Age Group (Initial Implant).

\begin{tabular}{|c|c|c|c|c|c|c|c|c|c|}
\hline \multirow[t]{2}{*}{ Year postimplant } & \multicolumn{3}{|c|}{ Adult patients } & \multicolumn{3}{|c|}{ Pediatric patients } & \multicolumn{3}{|c|}{ Total } \\
\hline & $\begin{array}{l}\text { No. of } \\
\text { interventions }\end{array}$ & $\begin{array}{l}\text { No. of } \\
\text { patients }\end{array}$ & $\begin{array}{l}\text { Intervention/ } \\
\text { patient }\end{array}$ & $\begin{array}{l}\text { No. of } \\
\text { interventions }\end{array}$ & $\begin{array}{l}\text { No. of } \\
\text { patients }\end{array}$ & $\begin{array}{l}\text { Intervention/ } \\
\text { patient }\end{array}$ & $\begin{array}{l}\text { No. of } \\
\text { interventions }\end{array}$ & $\begin{array}{l}\text { No. of } \\
\text { patients }\end{array}$ & $\begin{array}{l}\text { Intervention/ } \\
\text { patient }\end{array}$ \\
\hline 0-1 & 83 & 738 & 0.112 & 30 & 224 & 0.134 & 113 & 962 & 0.117 \\
\hline $1-2$ & 25 & 592 & 0.042 & 18 & 215 & 0.084 & 43 & 807 & 0.053 \\
\hline $2-3$ & 25 & 537 & 0.047 & 14 & 216 & 0.065 & 39 & 753 & 0.052 \\
\hline $3-4$ & 28 & 470 & 0.060 & 14 & 192 & 0.073 & 42 & 662 & 0.063 \\
\hline $4-5$ & 22 & 391 & 0.056 & 17 & 177 & 0.096 & 39 & 568 & 0.069 \\
\hline $5-6$ & 22 & 332 & 0.066 & 13 & 149 & 0.087 & 35 & 481 & 0.073 \\
\hline $6-7$ & 30 & 275 & 0.109 & 33 & 125 & 0.264 & 63 & 400 & 0.158 \\
\hline $7-8$ & 12 & 207 & 0.058 & 1 & 103 & 0.010 & 13 & 310 & 0.042 \\
\hline $8-9$ & 5 & 152 & 0.033 & 8 & 83 & 0.096 & 13 & 235 & 0.055 \\
\hline $9-10$ & 5 & 107 & 0.047 & 3 & 70 & 0.043 & 8 & 177 & 0.045 \\
\hline
\end{tabular}



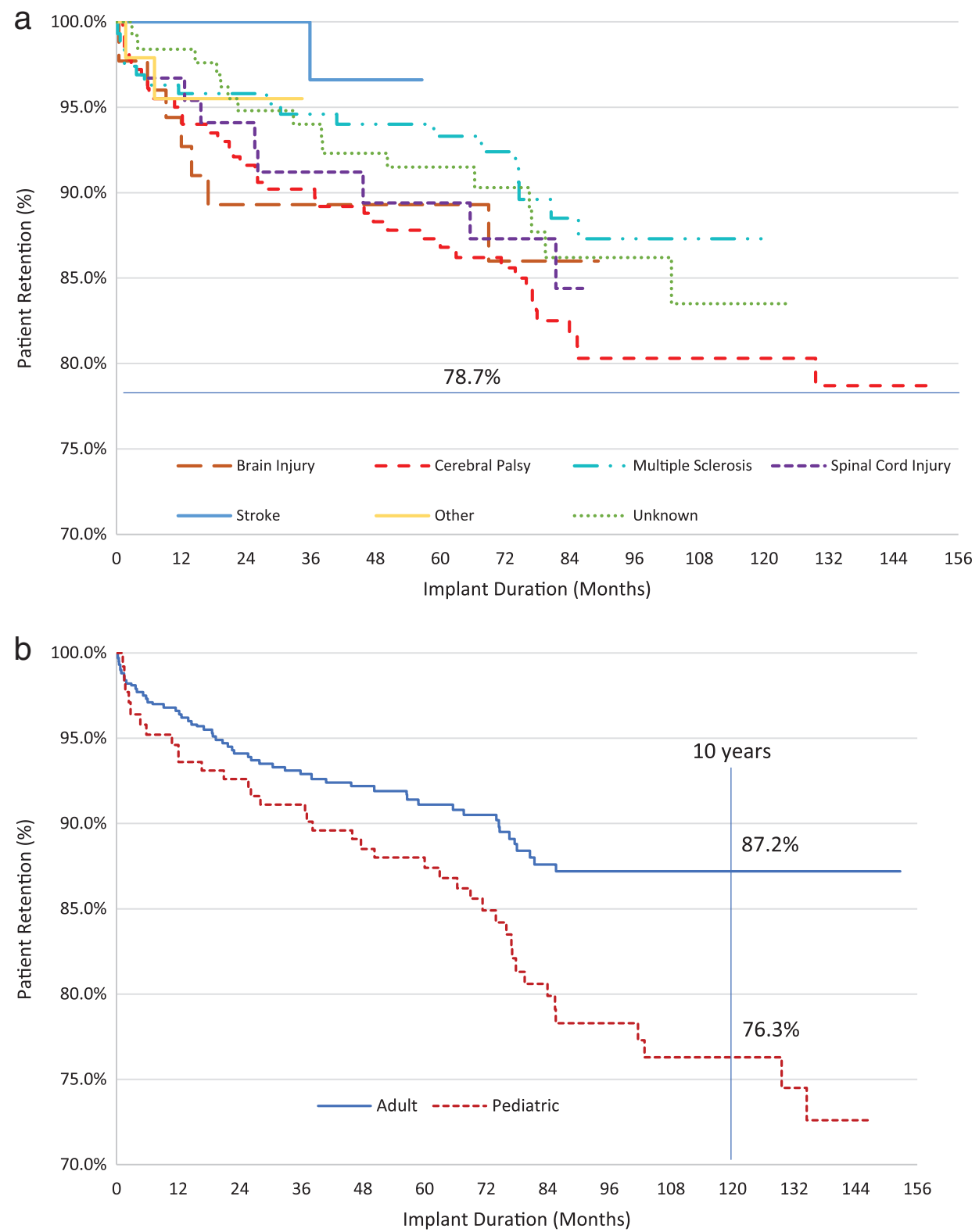

Figure 2. Patient survival from therapy-related study exit: a. By indication; b. by age category. [Color figure can be viewed at wileyonlinelibrary.com]

spastic hypertonia and muscle tone, a positive trend toward greater functional ability favored ITB. AEs (collected regardless of therapy relatedness) were more frequently reported in the ITB group, where $24 / 25(96 \%)$ of subjects reported 149 events, compared with CMM, where $22 / 35(63 \%)$ subjects reported 77 events. In the ITB group, approximately half of all events (69 of 149) occurred during the initial six-week dose-titration period. Reported events were consistent with the known safety profile of ITB. Four events in four patients randomized to the ITB arm were classified as serious adverse device events (device dislocation, device occlusion, implant-site infection and intracranial hypotension), and three led to device revision.

These randomized controlled trials and prospective case series include a limited number of participants enrolled and managed within tight study protocols for limited duration, and thus run the risk of under- or overestimating some therapy-related AEs. Realworld evidence from a large registry provides additional data to offer patients and clinicians a clearer assessment of the safety profile of a therapy. The PSR is the largest implanted drug delivery system registry in the world, with global and diverse clinical-site participation that offers a unique and pragmatic perspective on long-term ITB and adds to the general applicability of reported outcomes. Although spasticity-specific clinical outcomes in the PSR may be limited, therapy retention and elective pump replacement may reflect overall patient satisfaction and continued commitment to ITB therapy. The registry also selects study sites that represent commitment for long-term follow-up of patients and therefore will likely reflect more experienced and optimal care and management of the device when compared with novice implant centers or centers that implant ITB occasionally. Therefore, our AE rate may underestimate the complication rate and overestimate satisfaction compared with implant sites and subjects reported elsewhere in the literature.

AEs within the PSR are collected prospectively with risk-based monitoring to ensure accuracy and completeness. The risks associated with ITB therapy and the SynchroMed infusion system have been well studied, with publications on individual patient cases or 
Table 3. Duration of Pump Implants With Normal Battery Depletion.

$\begin{array}{llll}\begin{array}{l}\text { Spasticity } \\ \text { etiology }\end{array} & \text { N } & \begin{array}{l}\text { Mean (SD) implant } \\ \text { duration (months) }\end{array} & \begin{array}{l}\text { Same day } \\ \text { replacement }\end{array} \\ \begin{array}{l}\text { Cerebral palsy } \\ \text { Multiple sclerosis }\end{array} & 168 & 72.1(7.7) & 165(98.2 \%) \\ \text { Spinal cord injury } & 50 & 71.6(8.9) & 120(100.0 \%) \\ \text { Brain injury } & 31 & 70.6(13.2) & 50(100.0 \%) \\ \text { Stroke } & 15 & 74.7(8.6) & 30(96.8 \%) \\ \text { Other } & 3 & 81.1(2.2) & 15(100.0 \%) \\ \text { Unknown } & 75 & 72.5(12.7) & 3(100.0 \%) \\ \text { Total } & 462 & 72.0(9.7) & 75(100.0 \%) \\ \text { SD }=\text { standard deviation. } & & 458(99.1 \%)\end{array}$

series reports (20-23) and as many as 359 patients (24). The 1743 patients enrolled in the PSR and their average 44.6 months and cumulative 6481 patient-years of follow-up provide a currently unmatched source of safety data. Although the individual rates of specific complications are important in assessing safety, presenting "surgical risk" with a therapy for which surgical revision is often required for $\mathrm{AE}$ resolution has more relevance to both the treating physician and patient. As presented here, the risk of surgical revision (number of interventions/ITB naïve patients followed) is highest (adult: 0.112, pediatric: 0.134, cumulative: 0.117 ) in the first year post-implant and then rises again in years of anticipated pump replacement. In nonimplant years, surgical revision rates remain low (adult: 0.060, pediatric: 0.073, cumulative: 0.063 ) relative to published rates and stable.

Safety can be further evaluated in this registry through the use of patient survival from therapy discontinuation-that is, the percentage of patients who remain on therapy at specific time points. Study exit (whether due to an adverse or device event or to inactive products) was considered registry discontinuation due to an unacceptable risk-benefit ratio. Therapy continued beyond 10 years for $>75 \%$ of pediatric and adult patients in this registry, supporting the assertion of a positive risk-benefit determination, and high patient acceptance for ITB therapy.

Finally, these data offer the opportunity to further assess the riskbenefit ratio of ITB, provided that elective pump replacements are accepted as a surrogate for continued patient satisfaction. For patients maintaining ITB therapy for the lifetime of the implanted pump, pump replacement discussions can begin as much as one year before the required replacement surgery. During this time, the patient, their caregivers, and the managing physician can fully weigh the known and understood therapy risks against actual benefits received. In contrast to the theoretical risks and benefits associated with the first pump implant, implanted patients understand the daily risks and concerns associated with ITB therapy and the benefits derived. Patients also progress through other therapeutic options before ITB therapy, giving them insight into other therapies, and often into the consequences of no spasticity treatment. Analysis of elective pump replacement is possible in those patients who have both remained active in the registry and progressed to pump EOS-approximately 7 years. Although only a subset of the 1743 enrolled patients are available for analysis in this cohort (364 initial implants and 98 replacement implants), the significance of 426 patients with long-term ITB therapy (average 72 months) available for analysis cannot be understated. That $99 \%$ of these patients (98.9\% of ITB naïve patients), following careful consideration, opt for therapy continuation indicates high patient satisfaction.

Death was a reason for discontinuation for 178 (15\%) patients in this cohort with 169 reported as unrelated to the device, therapy or procedure and nine deaths of unknown or unreported relation to device/therapy. Two of these nine deaths involved pump pocket infections in immunocompromised patients, which are not classified as device related: one patient treated with ITB for traumatic brain injury-related spasticity experienced a pump pocket infection within 1 month of implantation that progressed to sepsis and resulted in death; the second patient, being treated for CP-related spasticity, experienced pump pocket erosion and an MSSA-positive infection 2 years post-implantation that resulted in death. A third patient death was associated with urinary tract infection and meningitis and reported as not device related, although system explant was undertaken. No literature providing mortality statistics specific to ITB therapy in patients with severe spasticity across all indications was identified, but research has shown that mortality in CP is the same with or without ITB treatment (25) and that infection risk is higher in the pediatric population compared to adults (26).

Registries such as the PSR are not without limitations. Physicians maintain their standard clinical practice and this lack of a uniform treatment limits the possibility of reproducing study results in other studies or within a clinical practice. Patient follow-up continues until a definitive therapy-related event occurs (e.g., therapy discontinuation or death), but patients may discontinue registry participation due to study site closure or to a transfer of care (e.g., pediatric patients aging up to adult management), limiting full follow-up. This study has more adult than pediatric spasticity patients, possibly skewing the cumulative results in favor of lower risk patients, whose bodies are no longer growing. The duration of this study ( 15 years) additionally spans changes in ITB therapy best practices. These limitations are balanced by the fact that site selection and physician participation include various practice levels, including academic medical centers and private practice. In addition, high data quality is expected as implanted patients maintain close contact with the prescribing physician to continue/discontinue therapy. Finally, we have used acceptance of pump replacement as a surrogate for patient satisfaction with ITB, but patients may possibly opt for a pump replacement as a routine, or for fear of baclofen withdrawal reactions.

\section{CONCLUSIONS}

The PSR provides the largest adult and pediatric patient cohort to date with results on the long-term safety and device performance of ITB in the treatment of severe spasticity of cerebral and spinal origin. Both low AE-related therapy discontinuation rates and high acceptance of surgical intervention for therapy continuation were observed. Although other unmeasured factors may contribute to continuation and satisfaction of ITB therapy, the patient/caregiver willingness to continue ITB and accept surgical and other therapy risks was extremely high.

\section{Acknowledgements}

The authors thank the physicians and centers that contributed data to the Product Surveillance Registry. Behzad Aalaei, Advanced Pain Management and Anesthesiology, P.C., IN, USA; Charles Edward Anderson, Desert Pain Medicine Group, CA, USA; Susan Apkon, Seattle Children's Hospital, WA, USA; Mark Barhorst, Center for Pain Recovery, PA, TX, USA; Francois Bethoux, Cleveland Clinic, OH, USA; Aaron Calodney, Precision Spine Care, TX, USA; Orlando Charry, Fairview Pain Management Center, MN, USA; Neal Coleman, Ball Memorial Hospital, IN, USA; Shrif Costandi, Cleveland Clinic, OH, USA; Michael Drass, Allegheny Pain 
Management PC, PA, USA; Stuart DuPen, Overlake Hospital Medical Center, WA, USA; Eric Grigsby, Spectrum Care Inc, CA, USA; Jon Hillyer, Peninsula Pain Clinic, WA, USA; John Huffman, Holy Cross Hospital (Silver Spring MD), MD, USA; Eduardo Ibarra, Clinica Manejo del Dolor, Puerto Rico; Frank Jordan, Pain Consultants of Tennessee, TN, USA; Brian Kahan, Center for Pain Medicine and Physiatric Rehab, MD, USA; Kathleen Kolaski, Wake Forest University Health Sciences/Baptist Medical Center/North Carolina Baptist Hospital, NC, USA; Donald Leslie, Shepherd Center Inc, GA, USA; John Loeser, University of Washington (UW) Medical Center, WA, USA; Bruce Massau, Pain Management Consortium of Ohio, $\mathrm{OH}$, USA; Alon Mogilner, North Shore University Hospital, NY, USA; Rosa Navarro, Michiana Anesthesia Care PC, IN, USA; Allison Oki, Primary Children's Hospital, UT, USA; Christophe Perruchoud, Hopital de Morges - Ensemble Hospitalier de la Cote, Switzerland; Fabian Piedimonte, Fundacion CENIT, Argentina; Stephen Pyles, Florida Pain, FL, USA; Richard Rauck, The Center for Clinical Research/ Carolina Pain Institute, NC, USA; Mark Romanoff, Southeast Pain Management Services, NC, USA; G. Sam Samarasinghe, Comprehensive Pain Management Centers, VA, USA; Mahendra Sanapati, Advanced Pain Care Clinic - Global Scientific, IN, USA; John Sasaki, CA, USA; David Schultz, Medical Advanced Pain Specialists (MAPS), MN, USA; Michael Sebahar, Coastal Pain Research, CA, USA; Andrew Seltzer, Pasadena Rehabilitation Institute, CA, USA; Thomas Silvestrini, Saint Mary's Duluth Clinic Health System, MN, USA; Angela Sinner, Gillette Children's Specialty Healthcare, MN, USA; Konstantin Slavin, The Board of Trustees of the University of Illinois, IL, USA; Kevin Smith, Integrated Pain Specialists of Southern California, CA, USA; Lisa J Stearns, Center for Pain and Supportive Care, AZ, USA; Onno P.M. Teernstra, Maastricht Universitair Medisch Centrum (MUMC), Netherlands; Tom Theys, UZ Leuven - Campus Gasthuisberg, Belgium; Ashish Udeshi, Florida Pain Institute Merritt Island, FL, USA; Angela Vallejo, Millennium Pain Center, IL, USA; Jan Vesper, Universitatsklinikum Dusseldorf, Germany; Kenneth Willis, Alabama Pain Center, AL, USA; Joel W. Winer, WellSpan Neurosurgery, PA, USA; Carlos Jaime Yepes, Clinica Las Americas, Colombia; Samuel Yue, MN Pain Center, MN, USA.

\section{Authorship Statements}

Dr. Schiess wrote the first draft and participated in statistical analysis execution, review and critique. Dr. Eldabe, Dr. Plunkett, Dr. Konrad, and Dr. Molus participated in statistical analysis execution, review and critique, and in writing review and critique. R. Spencer participated in statistical analysis review and critique, and in writing review and critique. K. Stromberg participated in statistical analysis design and execution. T. Weaver participated in research conception, organization and execution, in statistical analysis review and critique, and in writing review and critique.

\section{How to Cite this Article:}

Schiess M.C., Eldabe S., Konrad P., Molus L., Spencer R., Stromberg K., Weaver T., Plunkett R. 2020. Intrathecal Baclofen for Severe Spasticity: Longitudinal Data From the Product Surveillance Registry.

Neuromodulation 2020; 23: 996-1002

\section{REFERENCES}

1. Ghai A, Garg N, Hooda S, Gupta T. Spasticity - pathogenesis, prevention and treatment strategies. Saudi J Anaesth 2013;7:453-460.

2. Ertzgaard P, Campo C, Calabrese A. Efficacy and safety of oral baclofen in the management of spasticity: a rationale for intrathecal baclofen. J Rehabil Med 2017;49:193-203.

3. National Health Service Commissioning Board. Clinical Commissioning Policy: Intrathecal Baclofen (ITB). London, England: Crown Publishers; 2013.

4. Narendran RC, Duarte RV, Valyi A et al. The need for and provision of intrathecal baclofen therapy for the management of spasticity in England: an assessment of the Hospital Episode Statistic database. BMJ Open 2015;5:e007517.

5. Saulino M, Guillemette S, Leier J, Hinnenthal J. Medical cost impact of intrathecal baclofen therapy for severe spasticity. Neuromodulation 2015;18:141-149. discussion 149.

6. Vidal J, Slof J, Serrano D, Marqués T, Kumru H, Benito-Penalva J. Costeffectiveness of intrathecal baclofen therapy in severe refractory non-focal disabling spasticity: a Spanish hospital perspective. Expert Rev Pharmacoecon Outcomes Res 2017:17:67-76

7. Lissovoy G, Matza LS, Green $\mathrm{H}$ et al. Cost-effectiveness of intrathecal baclofen therapy for the treatment of severe spasticity associated with cerebral palsy. $J$ Child Neurol 2007:22:49-59.

8. Konrad PE, Huffman JM, Stearns LM et al. Intrathecal drug delivery systems (IDDS): The Implantable Systems Performance Registry (ISPR). Neuromodulation 2016;19:848-856.

9. Coffey JR, Cahill D, Steers W et al. Intrathecal baclofen for intractable spasticity of spinal origin: results of a long-term multicenter study. J Neurosurg 1993;78: 226-232.

10. Ordia Jl, Fischer E, Adamski E, Spatz EL. Chronic intrathecal delivery of baclofen by a programmable pump for the treatment of severe spasticity. $J$ Neurosurg 1996;85:452-457.

11. Albright AL, Gilmartin R, Swift D, Krach LE, Ivanhoe CB, McLaughlin JF. Long-term intrathecal baclofen therapy for severe spasticity of cerebral origin. $J$ Neurosurg 2003;98:291-295.

12. Meythaler JM, McCary A, Hadley MN. Prospective assessment of continuous intrathecal infusion of baclofen for spasticity caused by acquired brain injury: a preliminary report. J Neurosurg 1997;87:415-419.

13. Ivanhoe $C B$, Francisco GE, McGuire JR et al. Intrathecal baclofen management of poststroke spastic hypertonia: implications for function and quality of life. Arch Phys Med Rehabil 2006;87:1509-1515.

14. Schiess MC, Oh IJ, Stimming EF et al. Prospective 12-month study of intrathecal baclofen therapy for poststroke spastic upper and lower extremity motor control and functional improvement. Neuromodulation 2011;14:38-45.

15. Creamer M, Cloud G, Kossmeh P et al. Intrathecal baclofen therapy versus conventional medical management for severe poststroke spasticity: results from a multicenter, randomized, controlled, open-label trial (SISTERS). J Neuro Neurosurg Psychiatr 2018;0:1-9.

16. Deer TR, Prager J, Levy R et al. Polyanalgesic Consensus Conference 2012: recommendations for the management of pain by intrathecal (intraspinal) drug delivery: report of an interdisciplinary expert panel. Neuromodulation 2012;15: 436-466.

17. Penn RD, Kroin JS. Continuous intrathecal baclofen for severe spasticity. Lancet 1985;2:125-127.

18. Delhaas EM, Beersen N, Redekop WK, Klazinga NS. Long-term outcomes of continuous intrathecal baclofen infusion for treatment of spasticity: a prospective multicenter follow-up study. Neuromodulation 2008;11:227-236.

19. Creedon SD, Dijkers MP, Hinderer SR. Intrathecal baclofen for severe spasticity: a meta-analysis. Int J Rehabil Health 1997;3:171-185.

20. Riordan J, Murphy P. Intrathecal pump: an abrupt intermittent pump failure. Neuromodulation. 2015;18:433-435.

21. Galica R, Hayek S, Veizi I et al. Sudden intrathecal drug delivery device motor stalls: a case series. Reg Anesth Pain Med 2016;42:135-139.

22. Awaad Y, Rizk T, Siddiqui I, Roosen N, McIntosh K, Waines GM. Complications of intrathecal baclofen pump: prevention and cure. ISRN Neurol 2012;2012:575168.

23. Chan DY, Chan SS, Chan EK et al. Blessing or burden? Long-term maintenance, complications and clinical outcome of intrathecal baclofen pumps. Surg Pract 2018;22:105-110.

24. Krach LE, Kriel RL, Day SM, Strauss DJ. Survival of individuals with cerebral palsy receiving continuous intrathecal baclofen treatment: a matched-cohort study. Dev Med Child Neurol 2010;52:672-676.

25. Vedanarayanan V, Vendanarayanan V, Parker C et al. Mortality in children with cerebral palsy treated with intrathecal baclofen pump [abstr]. Neurology 2015; 85:14.

26. Fjelstad AB, Hommelstad J, Sorteberg A. Infections related to intrathecal baclofen therapy in children and adults: frequency and risk factors. I Neurosurg Pediatr 2009;4:487-493. 